\title{
Improving Software Middleboxes and Datacenter Task Schedulers
}

\author{
Hugo Sadok, Miguel Elias M. Campista, Luís Henrique M. K. Costa* \\ Universidade Federal do Rio de Janeiro - GTA/PEE/COPPE \\ \{sadok, miguel, luish\}@gta.ufrj.br
}

\begin{abstract}
Shared systems have contributed to the popularity of many technologies. However, these systems often confront a common challenge: to ensure that resources are fairly divided without compromising utilization efficiency. In this master's thesis we look at this problem in two distinct systems-software middleboxes and datacenter task schedulers. We first present Sprayer, a system that uses packet spraying to load balance packets to cores in software middleboxes. Our design eliminates the imbalance problems of per-flow solutions and addresses the new challenges of handling shared flow states that come with packet spraying. Then, we present Stateful Dominant Resource Fairness (SDRF), a task scheduling policy for datacenters that looks at past allocations and enforces fairness in the long run. SDRF reduces users' waiting time on average and improves fairness by increasing the number of completed tasks for users with lower demands, with small impact on high-demand users.
\end{abstract}

\section{Motivation and Problem Statement}

Over the last decades, shared systems have contributed to the popularity of many technologies. From Operating Systems to the Internet, they have all brought significant cost savings by allowing the underlying infrastructure to be shared. A common challenge in these systems is to ensure that resources are fairly divided without compromising utilization efficiency. This tradeoff between efficiency and fairness presents itself in a variety of ways and in different levels of system design. In this master's thesis we present ideas that improve both efficiency and fairness in two popular shared systems: software middleboxes and datacenter task schedulers. In the following subsections we describe the two problems we tackle.

\subsection{Inefficient Use of Multiple Cores in Software Middleboxes}

Today middleboxes are a primary component of both enterprise and Internet provider networks [Sekar et al. 2012]. Middleboxes allow network operators to deploy a wide range of network functions (NFs), such as Network Address Translators (NATs), firewalls, and load balancers. Yet, the cost and lack of flexibility of purpose-built hardware middleboxes are pushing operators to software running on commodity servers. Moving to software, however, does not come for free. Software middleboxes have significant overhead

\footnotetext{
*The content of this paper is partially adapted from our previously published works [Sadok et al. 2018a, Sadok et al. 2018b] as well as from the master's thesis [Barreto 2018]. Another version of this paper was also published at CTD@ SBRC 2019. This study was financed in part by the Coordenação de Aperfeiçoamento de Pessoal de Nível Superior - Brasil (CAPES) - Finance Code 001, CNPq, FAPERJ, and FAPESP grants \#15/24494-8 and \#15/24490-2.
} 
and often need to use multiple CPU cores - or even multiple hosts-to achieve line rates. Moreover, the rapid increase of network link capacities only exacerbates this need.

When using multiple cores, middleboxes must determine which core to direct packets to. Today, this is done using Receive-Side Scaling (RSS). RSS is a feature of multi-queue network interface controllers (NICs) that directs packets to different cores using a hash of the five-tuple. Doing so, all packets from the same flow end up in the same core. The reasons for coupling packets from the same flow are twofold. First, processing same-flow packets sequentially avoids packet reordering. Second, having sameflow packets processed in the same core simplifies flow state handling. RSS, however, has significant shortcomings. It is inefficient, since it cannot use all the available cores when the number of concurrent flows is small-which happens frequently in real workloads [Barreto 2018, §3.1]. Moreover, since RSS directs flows to cores using a hash of the five-tuple, hash collisions cause asymmetry in flow distribution. ${ }^{1}$ This results in unfairness even with a larger number of flows [Barreto 2018, §3.4]. In Section 2 we look at this problem and make a case for a natural alternative: that middleboxes should direct packets to cores at a finer granularity. We present a system that uses packet spraying to direct packets to cores in software middleboxes and addresses the new challenges of handling shared flow state that come with this new approach.

\subsection{Long-Term Unfairness in Datacenter Task Schedulers}

Modern datacenters are often shared by users with heterogeneous resource constraints [Reiss et al. 2012]. The amount of resources given to each user directly impacts the system performance from both fairness and efficiency standpoints. In single-resource systems, max-min fairness is the most widely used and studied allocation policy. The main idea is to maximize the minimum allocation a user receives. It was originally proposed to ensure a fair share of link capacity for every flow in a network. Since then, maxmin has been applied to a variety of individual resource types, including CPU, memory, and I/O [Ghodsi et al. 2011]. Nevertheless, datacenters need to allocate multiple resource types at the same time (such as CPU and memory) and max-min is unable to ensure fairness [Ghodsi et al. 2011].

In a datacenter environment, users often have heterogeneous demands and dynamic workloads [Reiss et al. 2012]. Different mechanisms have been proposed to address the multi-resource allocation, most notably, Dominant Resource Fairness (DRF) [Ghodsi et al. 2011]. DRF generalizes max-min to the multi-resource setting, by giving users an equal share of their mostly demanded resource-their dominant resource. Using this approach, DRF achieves several desirable properties. Despite the extensive literature on fair allocation, most allocation policies focus only on instantaneous, or short term, fairness, ensuring that users receive an equal share of the resources regardless of their past behaviors. DRF is no exception, it guarantees fairness only when users' demands remain constant. In practice, however, users' workloads are quite dynamic [Reiss et al. 2012] and ignoring this fact leads to sub-optimal allocations and unfairness in the long run. In Section 3 we propose a mechanism that extends DRF to consider past allocations. We show that this mechanism ensures fairness in the long run and reduces user's waiting time on average.

\footnotetext{
${ }^{1}$ Even when the number of cores is comparable to the number of flows, hash collisions happen with high probability due to the birthday problem.
} 


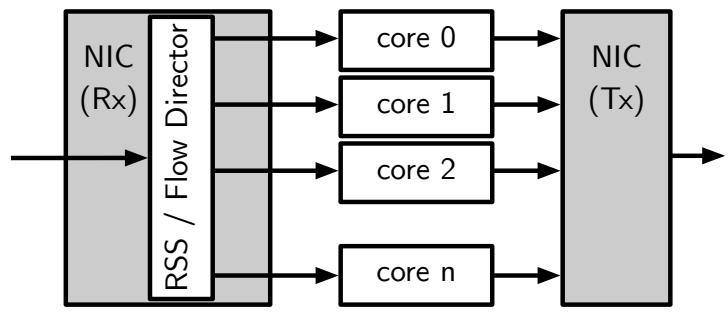

Figure 1. Software middlebox: the NIC can direct packets to cores using either RSS or Flow Director. Both send all packets from the same flow to the same core.

\begin{tabular}{|l|c|c|c|c|}
\hline \multirow{2}{*}{ NF } & \multirow{2}{*}{ State } & \multirow{2}{*}{ Scope } & \multicolumn{2}{|c|}{ Access Pattern } \\
\cline { 4 - 5 } & & packet & flow \\
\hline $\begin{array}{l}\text { NAT, } \\
\text { IPv4 to IPv6 }\end{array}$ & Flow map & Per-flow & R & RW \\
\cline { 2 - 5 } & Pool of IPs/ports & Global & - & RW \\
\hline Firewall & Connection context & Per-flow & R & RW \\
\hline \multirow{2}{*}{$\begin{array}{l}\text { Load } \\
\text { Balancer }\end{array}$} & Flow-server map & Per-flow & R & RW \\
\cline { 2 - 5 } & Pool of servers & Global & - & RW \\
\cline { 2 - 5 } & Statistics & Global & RW & - \\
\hline $\begin{array}{l}\text { Traffic } \\
\text { Monitor }\end{array}$ & Connection context & Per-flow & - & RW \\
\cline { 2 - 5 } $\begin{array}{l}\text { Redundancy } \\
\text { Elimination }\end{array}$ & Statistics & Global & RW & - \\
\hline DPI & Aacket cache & Global & RW & - \\
\hline
\end{tabular}

Table 1. Example of state scope and access pattern of some popular stateful NFs. Most NFs only update flow state when connections start or finish.

\section{Sprayer}

To solve the imbalance problems caused by hashing flows to cores in software middleboxes, we take inspiration from a similar problem in a different domain: datacenter networks. Traditionally, datacenter networks use Equal Cost Multi-Path (ECMP) to direct packets to different paths. Like RSS, ECMP directs all packets from the same flow to the same path and, as such, has similar shortcomings. This observation has led recent works to consider load-balancing packets ignoring their flows. This approach, known as packet spraying, introduces reordering but, because datacenter networks have paths with low and very similar latencies, the amount of reordering is not enough to significantly harm TCP. In the first part of the master's thesis we propose Sprayer, a system that allows the development of network functions using packet spraying. There are two main challenges in the design of Sprayer: spraying packets using existing NICs and handling flow states.

\subsection{Spraying Packets Using Existing NICs}

At first glance, it may seem impossible to spray packets using commodity NICs, since they do not offer this functionality (see Figure 1). We circumvent this limitation using Flow Director, a functionality found in many NICs, designed to associate specific sets of flows to cores. To do this, Flow Director allows us to specify rules using header fields. We use this capability in an unconventional manner: instead of matching sets of flows, we configure rules to direct packets to cores based on checksum field of the TCP header. Since this field looks random, TCP packets are uniformly distributed across cores, regardless of their flows. Non-TCP packets fail to match any rules and fall back to RSS. This avoids the potential problems packet reordering causes to some UDP applications.

\subsection{Handling Flow States}

When we send all the packets from the same TCP connection to the same core, we benefit from having partitionable flow states, which ensures that each core only has to keep state for its flows. Partitionable state is desirable, as it avoids the penalty of enforcing cache coherence, as well as the use of synchronization primitives. When we use packet spraying, packets from the same flow may go to different cores and this property no longer holds. What we observe, however, is that we get similar benefits if we instead provide writing 
partition. As long as we guarantee that each flow state can only be modified by a single core, we avoid the use of locks and significantly reduce cache invalidations.

To ensure writing partition, we depart from the observation that most NFs only change flow state when TCP connections start or finish. Table 1 shows the scope (perflow or global state) and access pattern (read or write at every packet or flow) for some popular stateful NFs. To leverage this observation, Sprayer makes a distinction between connection packets and regular packets. Connection packets are those that have potential to modify TCP state (those flagged with SYN, FIN, or RST), while regular packets are all the others. Sprayer ensures writing partition for flow states by making sure that all connection packets from the same TCP connection are processed by the same core. ${ }^{2}$

\subsection{Results}

We implemented Sprayer on top of DPDK ${ }^{3}$ and conducted experiments to understand how effective Sprayer is in comparison to RSS. Similarly to the datacenter observations, we find that the low difference in delay between packets processed in different cores is not enough to significantly impair TCP performance. Moreover, we observe that the overall TCP throughput remains consistent for both low and high number of concurrent flows. Therefore, for the typical number of concurrent flows found in real workloads, Sprayer greatly improves TCP throughput, compared to RSS. Furthermore, we show that Sprayer also improves fairness, even with a higher number of flows. For a more detailed description of the results, refer to the master's thesis [Barreto 2018, §3.4].

\section{Stateful Dominant Resource Fairness}

In the second part, we introduce Stateful Dominant Resource Fairness (SDRF), an extension of the DRF mechanism that accounts for the past behavior of users and improves fairness in the long run. The key idea is to make users with lower average usage have priority over users with higher average usage. When scheduling tasks, SDRF ensures that users that only sporadically use the system have their tasks scheduled faster than users with continuous high usage. The intuition for SDRF is that when users use more resources than their rightful share of the system, they commit to use less in the future if another user needs. SDRF tracks users' commitments and ensures that whenever system resources are insufficient, commitments are honored. We calculate commitments using an exponential moving average, avoiding the need to store the allocation history.

To illustrate the benefits of considering the past in an allocation, consider an example with two users sharing a system. To simplify, assume both users have the same dominant resource (e.g., CPU). User A is eager for resources and continuously submits a huge amount of tasks. In contrast, user B only uses the system sporadically. If we use DRF, whenever user B has a usage spike, both users get access to the same amount of resources - even though user B does not use the system as much as user A (see top of Figure 2). Alternatively, if we use SDRF, user A's commitment ensures that user B has

\footnotetext{
${ }^{2}$ Note that the only NF on Table 1 that needs to update flow state for every packet is Deep Packet Inspection (DPI), which means that Sprayer cannot be used to implement it. Also note that some NFs need to update global state for every packet. This problem affects traditional flow-based approaches as well as Sprayer. Fortunately, for some types of global states, such as statistics, looser consistency is often tolerable, which helps to reduce its impact.

${ }^{3}$ Data Plane Development Kit: https : / / www . dpdk . org/.
} 


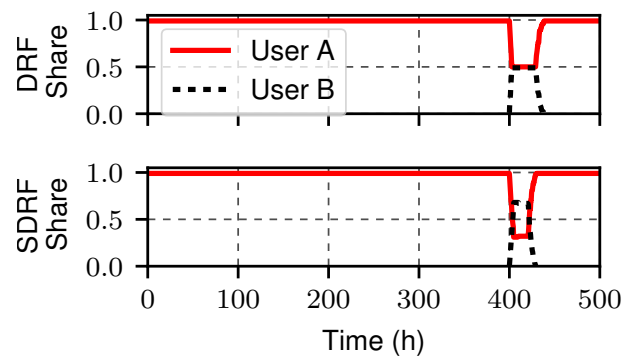

Figure 2. Share of dominant resource through time for two users when using DRF or SDRF.

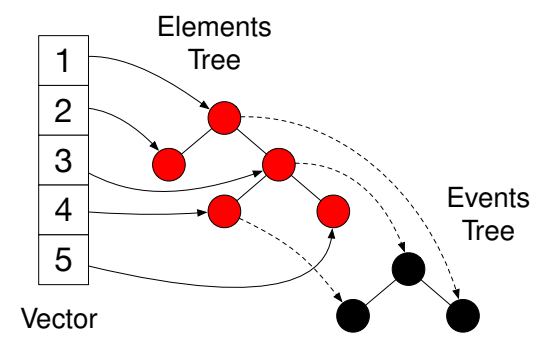

Figure 3. Illustration of a live tree with its data structures.

access to a greater share of resources. Because of it, user B is able to complete her workload faster (see bottom of Figure 2). Also notice that, with SDRF, system's resources go back to user A sooner than if we were using DRF, which ends up causing very little impact in user A's workload. When we ensure long-term fairness, we are able to improve the allocation for users with lower demand with little impact on users with higher demand.

We conduct a thorough evaluation of SDRF and show that it retains the fundamental properties of DRF. SDRF is strategyproof, as users cannot improve their allocation by lying to the mechanism. SDRF provides sharing incentives, as no user is better off if resources are equally partitioned. Moreover, SDRF is Pareto efficient, as no user can have her allocation improved without decreasing another user's allocation. The proof of all properties can be obtained in the master's thesis [Barreto 2018, §4.8].

\subsection{Practical Considerations}

Besides having desirable theoretical properties, a useful task scheduling policy must be efficiently implementable. In peak hours a scheduler may need to make hundreds of task placement decisions per second [Reiss et al. 2012]. While DRF can be efficiently implemented using a priority queue that determines which user has the highest allocation priority, when we consider the past, allocation priorities may change at any instant and the implementation cannot benefit from a priority queue. We mitigate this problem-being able to implement SDRF efficiently-introducing live tree, a data structure that keeps elements with predictable time-varying priorities sorted.

The key idea of a live tree is to focus on position-change events, instead of element priorities. When priorities follow a continuous function, elements change position whenever their priorities intersect. A live tree always has a current time associated with it and for this current time, it guarantees that elements are sorted. When the current time is updated, instead of updating every element priority, we see if any position-change event happened from the last update to the current time. Figure 3 depicts a live tree: it is composed of two red-black trees and an array. One tree is the elements tree, since it keeps elements sorted by priority, while the other is the events tree, since it tracks position-change events sorted by their time. The array is used for element lookup. In the master's thesis we describe live tree's operations in detail and provide their worst-case time complexity.

\subsection{Results}

To understand how SDRF performs under real workloads and how it compares to DRF, we implemented a discrete-event simulator and fed it with Google cluster traces. These 
traces contain 30 million tasks (from either Google services or engineers) over a onemonth period. Our results show that SDRF reduces the average time users wait for their tasks to be scheduled. Moreover, it increases the number of completed tasks for users with lower demands, with negligible impact on high-demand users. We also use the simulations to evaluate the performance of live tree, concluding that SDRF can be efficiently implemented in practice. For a more detailed description of the results, see the master's thesis [Barreto 2018, §4.5].

\section{Impact}

As a result of this master's thesis we have published 3 conference papers-including a publication at ACM HotNets - and presented a poster at USENIX NSDI. ACM HotNets and USENIX NSDI are two of the most important and selective venues in the field of Computer Networks. The list of works follows:

1. H. Sadok, M. E. M. Campista, L. H. M. K. Costa. "A Case for Spraying Packets in Software Middleboxes." In ACM HotNets, pp. 127-133, Nov. 2018. Qualis A1.

2. H. Sadok, M. E. M. Campista, L. H. M. K. Costa. "O Passado Também Importa: Um Mecanismo de Alocação Justa de Múltiplos Tipos de Recursos ao Longo do Tempo.” In SBRC, May 2018. Qualis B2.

3. H. Sadok, M. E. M. Campista, L. H. M. K. Costa. "Um Mecanismo para Compartilhamento de Recursos em Nuvens Colaborativas Baseado na Credibilidade dos Usuários." In SBRC, pp. 458-471, May 2017. Qualis B2.

4. H. Sadok, M. E. M. Campista, L. H. M. K. Costa. "Per-Packet Load Balancing for Multi-Core Middleboxes." Poster in USENIX NSDI, Apr. 2018. Qualis A1.

Moreover, in the context of this master's thesis, we also co-authored the following journal paper:

5. R. S. Couto, H. Sadok, P. Cruz, F. F. Silva, T. Sciammarella, M. E. M. Campista, L. H. M. K. Costa, P. B. Velloso, M. G. Rubinstein. "Building an IaaS Cloud with Droplets: a Collaborative Experience with OpenStack." In Journal of Network and Computer Applications, vol. 117, pp. 59-71, Sep. 2018. Qualis A2 (Impact Factor: 3.991).

Besides the above publications we have open sourced our task-scheduling simulator as well as our implementation of SDRF and live tree. ${ }^{4}$ We also plan to release Sprayer's source code soon.

\section{References}

Barreto, H. F. S. S. M. (2018). Improving software middleboxes and datacenter task schedulers. Master's thesis, Universidade Federal do Rio de Janeiro.

Ghodsi, A., Zaharia, M., Hindman, B., Konwinski, A., Shenker, S., and Stoica, I. (2011). Dominant resource fairness: Fair allocation of multiple resource types. In USENIX NSDI.

Reiss, C., Tumanov, A., Ganger, G. R., Katz, R. H., and Kozuch, M. A. (2012). Heterogeneity and dynamicity of clouds at scale: Google trace analysis. In $A C M$ SoCC.

Sadok, H., Campista, M. E. M., and Costa, L. H. M. K. (2018a). A case for spraying packets in software middleboxes. In ACM HotNets.

Sadok, H., Campista, M. E. M., and Costa, L. H. M. K. (2018b). O passado também importa: Um mecanismo de alocação justa de múltiplos tipos de recursos ao longo do tempo. In $S B R C$.

Sekar, V., Egi, N., Ratnasamy, S., Reiter, M. K., and Shi, G. (2012). Design and implementation of a consolidated middlebox architecture. In USENIX NSDI.

\footnotetext{
${ }^{4}$ https://github.com/hsadok/sdrf
} 